therapy (CBT) and interpersonal psychotherapy (IPT) in adults with major depression.

Background: Very little is known about the patient characteristics associated with response to CBT and IPT. Certain patient characteristics may be general prognostic indicators of response, irrespective of therapy type; however, there may also be other specific patient characteristics that are indicators of response to a specific treatment type (CBT vs. IPT).

Methods: After providing informed written consent, 177 adults with major depression (DSM-IV) were randomized to receive 16 weekly sessions of either CBT or IPT. Patient characteristics were assessed prior to randomization. Outcome at the end of the weekly sessions was defined as percentage improvement on the clinician-rated Montgomery Asberg Depression Rating Scale (MADRS), which takes account of initial depression severity.

Results: Factors associated with greater percentage improvement on the MADRS over the course of therapy included receiving CBT, having a single episode of depression and a perception that the therapy (irrespective of treatment type) was logical. Individuals who had comorbid personality disorder symptomatology had higher per cent improvement over the course of treatment if they received CBT (60\%) rather than IPT (30\%).

Conclusions: Despite examination of a number of potential predictors of differential response to CBT and IPT, few were significant. However, this study suggests that CBT is a better treatment option for depressed patients with comorbid personality disorder than IPT.

\section{White matter hyperintensities: lateralization effects}

\section{N Cherbuin', K Anstey², W Wen ${ }^{3,4}$, P Sachdev 4 \\ ${ }^{1}$ Australian National University; ${ }^{2}$ Ageing Research Unit, Australian National University; ${ }^{3}$ Neuropsychiatric Institute (NPI), Prince of Wales Hospital; \\ ${ }^{4}$ School of Psychiatry, University of New South Wales, Sydney, Australia}

Background: The presence of white matter hyperintensities (WMHs) on magnetic resonance imaging (MRI) scans is associated with age. However, their functional significance is controversial with some studies reporting significant associations of WMHs volume with cognitive decline, while others have not found such an association. Because the human brain is anatomically and functionally lateralized, one potential confound in this area of research is an effect of WMHs lateralization in the left and right cerebral hemispheres and their varying intra- and interhemispheric transfer consequences. The present study investigated the relationship between WMHs lateralization and corpus callosum (CC) measures and fine motor and cognitive performance.
Methods: A subsample of 478 individuals aged 60-64 years from a larger community sample were assessed for fine motor control (Purdue Pegboard), global cognitive function, episodic memory and working memory. Subjects also underwent brain MRI scans. WMHs on T2-weighted FLAIR MRI scans were assessed using an automated procedure. CC areas were hand traced.

Results: WMHs were significantly more lateralized to the left in the frontal, temporal and parietal lobes, and lateralized to the right in the occipital lobe. Periventricular and whole hemisphere WMHs were not lateralized. WMHs lateralization was significantly associated with CC areas, hippocampal laterlization and fine motor control. These associations were modulated by the location of WMHs in each hemisphere and by sex.

Conclusion: The lateralization of WMHs is an important factor to consider in the interpretation of their association with callosal structure and behavioural measures of motor control and cognition.

\section{Models of health care delivery for depression}

\section{H Christensen}

Centre for Mental Health Research, Australian National University, Canberra, Australia

Background: A range of models exist for the delivery of depression services in primary care and the community. The efficacy of the following approaches was assessed: passive education campaigns, telephone and Internet interventions, schools programs, general practice (education, care management, enhanced care, selfhelp in practice) and community programs linked to general practice.

Method: Systematic review.

Results: There is evidence for the effectiveness of schools programs, Internet and telephone interventions, care management, enhanced care and some community programs. Evidence is weaker or inconclusive for passive education, education directed at GPs, pharmacist interventions and bulletin boards.

\section{The Illness Management and Recovery Program: an international effectiveness study \\ R Clancy', D Clancy ${ }^{1,2}$, K Mueser ${ }^{3}$, V Carr', T Lewin ${ }^{1,4}$ M Hazelton ${ }^{5}$, K Sly ${ }^{1}$}

${ }^{1}$ Centre for Mental Health Studies, Newcastle; ${ }^{2}$ Newcastle Mental Health Service; ${ }^{3}$ New Hampshire-Dartmouth Psychiatric Research Centre, USA; ${ }^{4}$ Hunter New England Mental Health; and ${ }^{5}$ University of Newcastle, Newcastle, Australia 
Background: Much research has been conducted to establish best practice in psychosocial interventions for mental illness. The challenge of ensuring that best practice is delivered to consumers has historically been left to program managers. This research contributes to the science of technology transfer: assessing the transferability, uptake and effectiveness of a comprehensive psychological intervention within routine mental health services.

TheIllnessManagementandRecoveryProgram(IMR) was developed as one of six evidence-based practices in the (US) National Evidence-based Practices Project. The paper reports on the implementation of the IMR in Newcastle, Australia, and outcomes from both Australian and US sites.

Methods: This study comprised a multicenter, international effectiveness study of a comprehensive, evidence-based psychosocial intervention for serious mental illness. The study centers included public mental health facilities in Newcastle and North Carolina. Rigorous fidelity and outcome measures were conducted at baseline, postintervention ( 9 months) and at follow-up (12 months).

Results: Retention in this comprehensive program was satisfactory $(76 \%)$. Statistically significant improvements were noted on a range of measures including the BSI, GAF, Coping Skills subscales, Knowledge of Illness, and the Recovery Assessment Scale.

Conclusions: The pilot data support the feasibility of implementing the IMR program in routine mental health settings. The findings suggest that participation in the program is associated with benefits in illness management, progress toward recovery and improved functioning. Individuals completing the IMR program reported significant improvements in hope, coping self-efficacy and distress-related to symptoms.

\section{Constructing longitudinal measures of maternal morbidity using a population health database: is use of the index birth record sufficient to identify obstetric risk factors for schizophrenia?}

\section{Croft, V Morgan, A Jablensky}

School of Psychiatry and Clinical Neurosciences, University of Western Australia, Perth, Australia

Background: Western Australia (WA) has extensive record-linked population health data collections. Midwives' records of WA births during 1980-1992 inclusive were linked and validated by the author to form sibships, which enable longitudinal family studies. Binary indicators of maternal morbidity were created using this database of prospectively collected birth data. A validated system, using case note reviews, for scoring obstetric complications as risk factors for psychiatric morbidity has been adapted for coding population data. This will be extended to 2001 births and include the mother's clinical history.

Methods: Women who have had only one birth during this period, who had multiple births or who had missing births in their sibship were excluded from analysis. Codes created reflect morbidity in the current pregnancy only, the one immediately prior, any prior pregnancy or, the current or any prior pregnancy.

Results: The database includes records of 472733 births to 249119 women with paternal data available for the majority of children. Of these, approximately $90000(36 \%)$ women were eligible for inclusion. Of the 67242 eligible women, who delivered during 1980-1992, severe underestimation of maternal conditions, affecting pregnancy, such as thyroid dysfunction, essential hypertension or epilepsy occurred when only index birth records were used.

Conclusions: Using data from the index birth only could underestimate the effects of maternal morbidity, as risk factors for outcomes such as schizophrenia, when compared with analysis of models that use longitudinal binary indicators of current and prior maternal illness.

\section{Altered tripartite synapse function: is this the problem in schizophrenia and bipolar disorder?}

\section{B Dean}

Rebecca L. Cooper Research Laboratories, Mental Health Research Institute, Melbourne, Australia

Schizophrenia and bipolar I disorder are serious psychiatric illnesses that affect approximately $4 \%$ of the Australian population. Both illnesses are thought to occur in individuals with a genetic predisposition after they encounter as yet unknown detrimental environmental factors. Postmortem central nervous system (CNS) studies have been undertaken to identify the combined impact of these two factors on both cellular and molecular cytoarchitecture. In particular, studies from our laboratories have shown changed levels of the glial-derived proteins apolipoprotein $\mathrm{E}$ and $\mathrm{D}$ in the cortex of subjects with schizophrenia and bipolar I disorder. In addition, we have shown changes in levels of $\mathrm{S} 100 \beta$, in the dorsolateral prefrontal cortex from subjects with bipolar II disorder. Significantly, it is known that levels of $\mathrm{S} 100 \beta$ are known to be potently regulated by serotonin through the serotonin $1 \mathrm{~A}$ receptor and receptors for apolipoprotein $\mathrm{E}$ are localized on neurons. Our data would therefore support the hypothesis that at least part of the pathology of schizophrenia and 